\title{
Prospective Metanalysis Within Complementary Medicine Research
}

Dear Editor:

It appears that medical research funders are reluctant to provide sufficient funding to allow fully powered randomized control trials (RCTs) within the field of complementary medicine (CM). Funders often state that there is no evidence base to make the decision concerning whether to award such a grant. Our research area is awash with promising, but not fully powered data or lower-level noncontrolled data. This lack of efficacy resulting from problems involving trying to apply the "gold standard" of RCTs results in health organizations not integrating $\mathrm{CM}$ into health care provision, regardless of patients' opinions.

Health organizations base many of their guidelines on what they regard as the top level of evidence (i.e., systematic reviews [SRs]), which are regarded as the gold standard of evidence by health care policymakers. SRs are thorough literature searches that incorporate methods (e.g., inclusion criteria) to prevent bias. Usually, SRs are based on the results from RCTs, such as the ones adopted by the Cochrane Collaboration, although there are moves to develop methodologies to systemize reviews of other, non-RCT articles, such being able to include cohort or qualitative papers. Examples of these articles would include as the work by the Campbell Collaboration or the Joanna Briggs Institute. However, even with these developments, policymakers are loath to supply therapies without support from fully powered RCT trials.

One benefit of SRs is that data from different trials reported in the included articles, if using a common measure of effect size, can be amalgamated using meta-analysis (MA). The results from an MA are more powerful estimates of the true effect size than those derived from a single trial because the MA is based on different trials, crossing geographical boundaries, populations, trial conduct, etc. As a MA pools data, it also results in a larger overall sample size because the MA includes populations from all of the included trials, therefore reaching higher statistical power to detect an effect. Although this type of analysis lends itself to complementary and alternative medicine (CAM) research, as many trials reported are small-scale, often, an MA cannot be conducted on the data obtained from included articles because such studies are often heterogeneous. One way that this can be overcome in CAM research is by collaborating using a prospective MA (PMA) design. In a PMA, trials are identified, evaluated, and determined prior to publication to establish eligibility for a post-trial MA, therefore, allowing prestandardization of information required for the post-hoc MA. The PMA is a way of building the CAM evidence base in the absence of being able to conduct large, full-scale trials.

Arguably, this approach takes more organizing and collaboration at a very early stage in research. This may take the form of liaising with other researchers within a discipline, nationally and internationally, to write the protocol initially, and having maintenance meetings to ensure a continuum of a study protocol. Any divergence from protocol for any reason would be discussed with the PMA group. However, in the absence of funders giving large grants to run fully powered RCTs to explore the efficacy of complementary therapies, I believe that this is the only way forward in forming an evidence base. A good resource for researchers who are interested in this methodology can be found here:

Ghersi D, Berlin J, Askie L on behalf of the Cochrane Prospective Meta-Analysis Methods Group. Prospective meta-analysis. In Higgins JPT, Green S, eds. Cochrane Handbook for Systematic Reviews of Interventions Version 5.0.0 [updated February 2008]. The Cochrane Collaboration 2008. Online document at: www.cochrane-handbook.org

Address correspondence to: Dawn-Marie Walker University of Nottingham Trent RDSU 14th Floor, the Tower University of Nottingham Nottingham, NG9 2GY United Kingdom

E-mail: Dawn-marie.Walker@nottongham.ac.uk 
ACM-2010-0529-Walker_1P.3d 10/25/10 6:18pm Page 2 


\section{AUTHOR QUERY FOR ACM-2010-0529-WALKER_1P}

AU1: Provide degree.

AU2: Provide degree. 